\title{
Azimuthal Characteristics on Blast Wave from a Cylindrical Charge - Small Scale Experiment -
}

\author{
Tomotaka Homae ${ }^{1, a^{*}}$, Yuta Sugitana ${ }^{2, \mathrm{~b}}$,Tohoharu Matsumura ${ }^{2, \mathrm{c}}$ and \\ Kunihiko Wakabayashi ${ }^{2, d}$ \\ ${ }^{1}$ Department of Maritime Technology, National Institute of Technology, Toyama College,1-2 \\ Ebie-neriya, Imizu, Toyama 933-0293 JAPAN \\ ${ }^{2}$ Research Institute of Science for Safety and Sustainability, National Institute of Advanced \\ Industrial Science and Technology, Central 5, 1-1-1 Higashi, Tsukuba, Ibaraki 305-8565 JAPAN \\ ahomae@nc-toyama.ac.jp, ${ }^{b}$ yuta.sugiyama@aist.go.jp, ct-matsumura@aist.go.jp, \\ dk-wakabayashi@aist.go.jp
}

Keywords: Explosion, Azimuth Angle, Non-Spherical Explosive, Blast Pressure, Impulse

Abstract. Azimuthal characteristics on blast wave from a cylindrical charge were experimentally investigated. A cylindrical PETN pellet, weight of $0.50 \mathrm{~g}$, was detonated on a large steel plate, which is a model of the ground surface. $0^{\circ}$ was defined as the direction of detonation, which was correspondence with the central symmetry axis of the cylindrical pellet. 12 pressure transducers were embedded in the steel plate to measure the pressure histories on the plate. The direction of the pellet and detonation was rotated every $30^{\circ}$ and the distribution of blast pressure histories around the explosive were obtained. The peak overpressure and impulse were high in the range from $30^{\circ}$ to $80^{\circ}$ compared with the standard explosion data in which the explosive was placed vertically for a two-dimensional axisymmetric explosion on the steel plate and detonated from the top of the explosive. On the contrary, these blast parameters were low in the range from $90^{\circ}$ to $130^{\circ}$. These blast pressures were not low in the direction of $180^{\circ}$. These findings are important for safety. The data will be compared with numerical simulations in future.

\section{Introduction}

Explosion of non-spherical explosive causes anisotropic blast wave. This anisotropic blast waves have been extensively studied for many decades. Especially, explosion of cylindrical explosive, which is relatively symmetric, have been studied both experimentally and numerically. R.A. Strehlow and W.E. Baker reviewed these studies in 1976 [1]. As the experimental technique and numerical analysis technique have been improving, many papers have been published until present [2]. It is noteworthy for safety that the blast wave in specific direction is reported to be strong in these papers.

The authors carried out indoor tabletop experiments for evaluating the blast wave, using explosive of $1 \mathrm{~g}$ scale, and the obtained data were examined by numerical analysis. The authors reported the blast wave mitigation or distribution in a couple of systems [3-6]. In this study, the authors applied the technique above to evaluate the blast wave distribution from a cylindrical explosive precisely. The experimental system was designed under consideration for numerical analysis.

\section{Experiment}

Test Explosives. A PETN pellet, weight of $0.50 \mathrm{~g}$, was used as a test explosive. Its length and diameter were both $7.5 \mathrm{~mm}$. It contained $5 \mathrm{wt} \%$ of carbon for forming. A specially designed electric detonator with $100 \mathrm{mg}$ lead azide was used as a detonator. Both the PETN pellet and the 
detonator were distributed by Showa Kinzoku Kogyo Co. Ltd. A spacer was used so as to the height of the center of the explosive was $0.18 \mathrm{~m} / \mathrm{kg}^{1 / 3}$. The scaled height was set up as the samescaled height of previous study [5] for comparison. The spacer was made of pasteboard and was rectangular block with the height of $10 \mathrm{~mm}$ and base of $7 \times 7 \mathrm{~mm}$. The lateral surface of the cylindrical pellet was fixed on the spacer using epoxy resin adhesive. $4 \mathrm{kV}$ was applied to initiate the detonator (test explosive) using a firing system (FS-43; Teledyne RISI, Inc.).

Ground Surface Model. A steel plate, length of $3510 \mathrm{~mm}$, width of $2200 \mathrm{~mm}$, and thickness of $10 \mathrm{~mm}$, was regarded as a ground surface. The test explosive with the spacer was fixed on the surface model using double-sided adhesive tape. Fig. 1 shows the configuration of the test explosive on the surface model. The direction of ignition is defined as $0^{\circ}$.

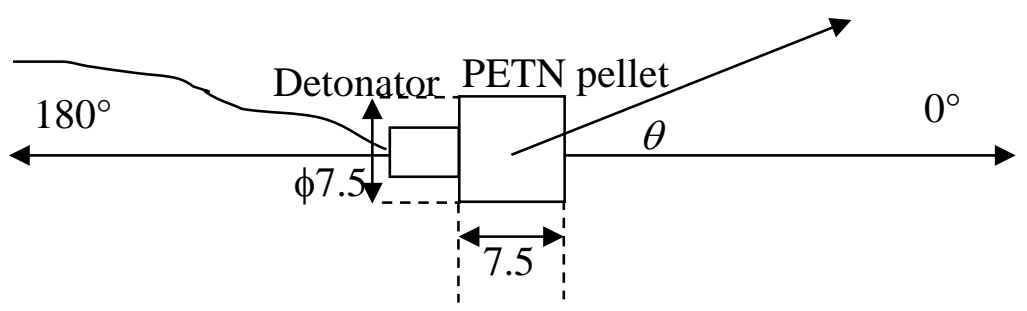

Fig. 1 Set up of test explosiveand definition of the angle.

Pressure Measurement.12 pressure transducers (113B28; PCB Piezotronics, Inc.) were used to measure the blast pressure at the ground model surface. The pressure transducers were set with the vibration isolator (GEL Tape; Taica Corporation) as the diaphragm of the transducer was flush with the ground surface model. The transducers were on three lines of every $10^{\circ}$. Then, the pressure histories of three directions, for example, $0^{\circ}, 10^{\circ}$ and $20^{\circ}$, were obtained in one explosion experiment. The distances from the center of the explosive were $400 \mathrm{~mm}, 800 \mathrm{~mm}$, $1200 \mathrm{~mm}$, and $1600 \mathrm{~mm}$, respectively. The corresponded Hopkinson scaled distance was from $5.1 \mathrm{~m} / \mathrm{kg}^{1 / 3}$ to $20.4 \mathrm{~m} / \mathrm{kg}^{1 / 3}$. The Hopkinson scaled distance was obtained the distance divided by cube root of the net weight of PETN, 95\% of the measured weight of the pellets. The output signals were recorded using a transient recorder (LTT184/8;LabortechnikTasler GmbH; sampling rate of $1.04 \mathrm{MHz}$ and resolution of 16 bits in this study) through an amplifier system (30510 and 30622; H-Tech Laboratories, Inc.).

Number of Experiments and Standard Experiments. The setup of explosive direction varied every $30^{\circ}$. Seven experiments gave one series of the data from $0^{\circ}$ to $180^{\circ}$. Two series of the data were acquired in this study. In addition to the experiments above, the standard data in which the explosive was placed vertically for a two-dimensional axisymmetric explosion on the steel plate and detonated from the top of the explosive were obtained for comparison.

\section{Results and Discussions}

The obtained pressure histories were fitted using spline functions. Then, peak overpressures and positive scaled impulse, just scaled impulse hereinafter, were determined. Fig. 2 (a) shows the relation between peak overpressure and azimuthal angle. The shown data is average of data by two experiments. The dotted line is the peak overpressure of the standard data. Fig. 2 (a) shows the absolute value, and the dependence on azimuth angle is not easy to understand at further points. Fig. 2(b) shows the peak overpressure ratio to the standard data. It is clearly seen that the explosive shape affected even at $20.4 \mathrm{~m} / \mathrm{kg}^{1 / 3}$. The peak overpressure is high from $30^{\circ}$ to $80^{\circ}$ (at $5.1 \mathrm{~m} / \mathrm{kg}^{1 / 3}$, from $30^{\circ}$ to $60^{\circ}$ ), compared with the standard data. The difference is from $10 \%$ to 
$25 \%$. On the contrary, It is low from $90^{\circ}$ to $120^{\circ}$. The peak overpressure was not low at the direction of $180^{\circ}$, that is, opposite to the direction of ignition.
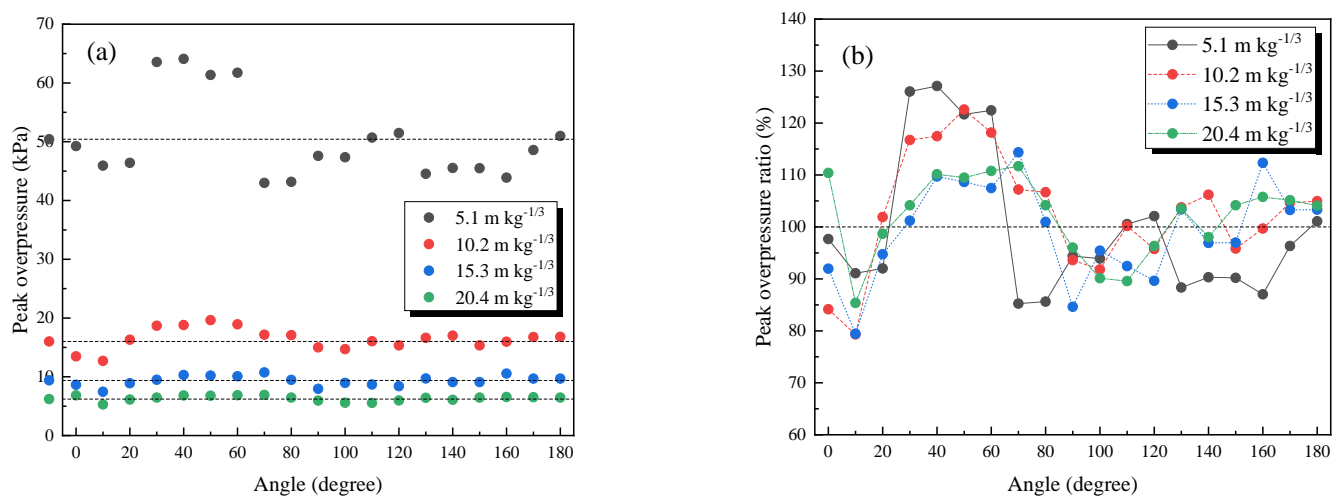

Fig. 2 (a) Dependence of peak overpressure on azimuthal angle. (b) Dependence of peak overpressure ratio to standard peak overpressure on azimuthal angle.

Fig. 3 (a) shows the dependence of scaled impulse on azimuthal angle. Fig. 3 (b) shows the dependence of the ratio on azimuthal angle. The tendency is similar to that of peak overpressure, although data scatter at $20.4 \mathrm{~m} / \mathrm{kg}^{1 / 3}$ is large.
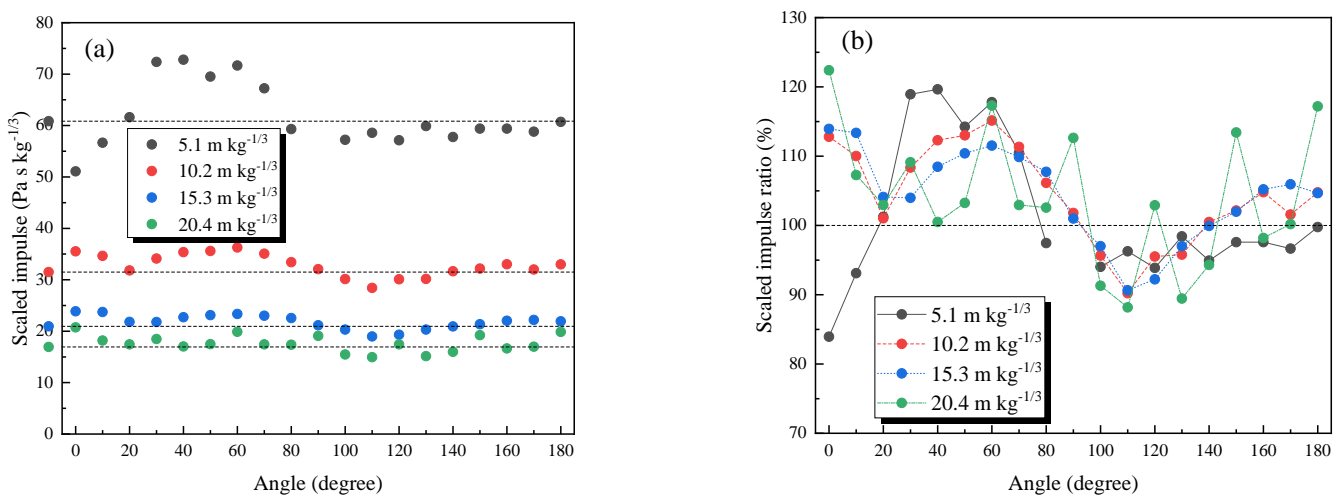

Fig. 3 Dependence of scaled impulseon azimuthal angle. (b) Dependence of scaled impulseratio to standard scaled impulse on azimuthal angle.

The relation between peak overpressure and the scaled distance on log-log plane was fitted using quadratic function. The peak overpressures of standard data at scaled distance of 8 $\mathrm{m} / \mathrm{kg}^{1 / 3}$ and $16 \mathrm{~m} / \mathrm{kg}^{1 / 3}$ were calculated using the fitted quadratic function. Scaled distance of 8 $\mathrm{m} / \mathrm{kg}^{1 / 3}$ and $16 \mathrm{~m} / \mathrm{kg}^{1 / 3}$ are regulated as safety distance in law in Japan. The calculated peak overpressures were $23.67 \mathrm{kPa}$ and $8.59 \mathrm{kPa}$, respectively. Then, the scaled distances, where the peak overpressures were $23.67 \mathrm{kPa}$ and $8.59 \mathrm{kPa}$, were determined for every direction (from $0^{\circ}$ to $180^{\circ}$ ). Same procedure was adopted to the scaled impulse. The scaled impulses at 8 $\mathrm{m} / \mathrm{kg}^{1 / 3}$ and $16 \mathrm{~m} / \mathrm{kg}^{1 / 3}$ were $39.12 \mathrm{~Pa} \mathrm{~s} / \mathrm{kg}^{1 / 3}$ and $20.63 \mathrm{~Pa} \mathrm{~s} / \mathrm{kg}^{1 / 3}$, respectively. Figs. 4 (a) and (b) show the isobaric line of peak overpressure and scaled impulse. The plots form $190^{\circ}$ to $350^{\circ}$ in the figure are just the mirror image of $170^{\circ}$ to $10^{\circ}$. 

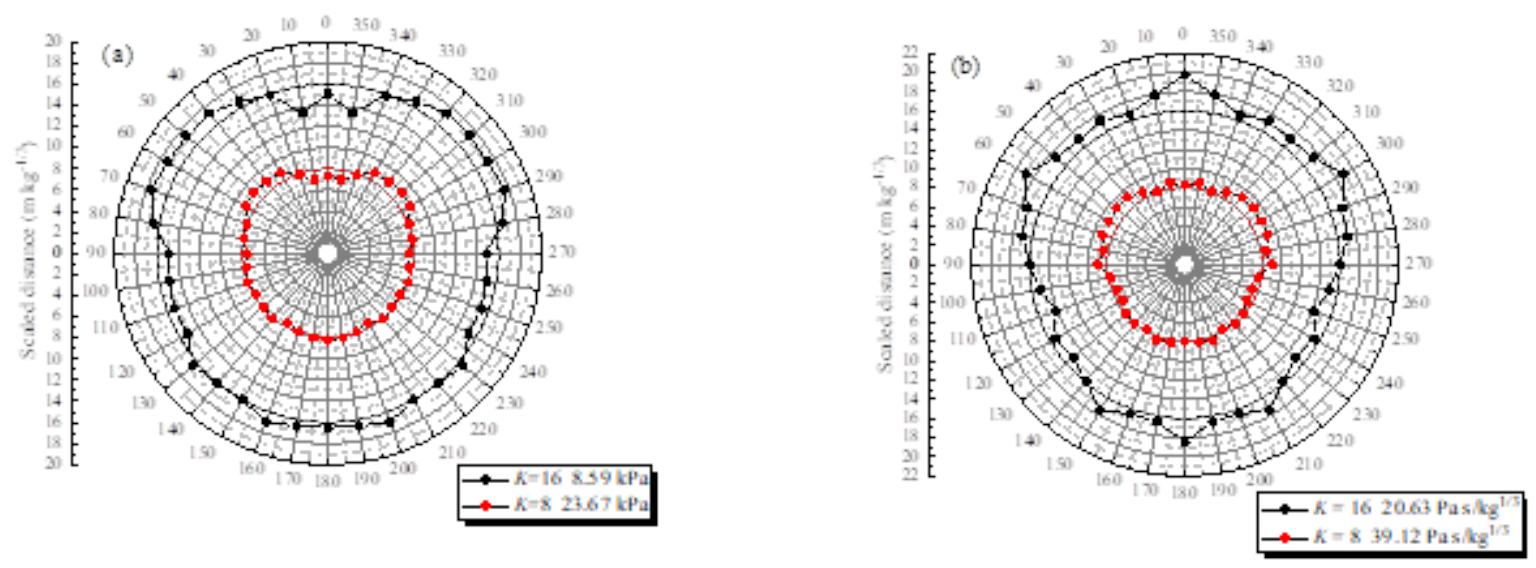

Fig. 4 Isobaric line of (a) peak overpressure and (b)scaled impulse.

Regarding to the peak overpressure, the isobaric line expanded, up to scaled distance of approximately $2 \mathrm{~m} / \mathrm{kg}^{1 / 3}$, at the direction from $30^{\circ}$ to $80^{\circ}$. This means that the peak overpressure is same as the standard data at scaled ditance of $16 \mathrm{~m} / \mathrm{kg}^{1 / 3}$, at scaled distance of $17 \mathrm{~m} / \mathrm{kg}^{1 / 3}$ or even $18 \mathrm{~m} / \mathrm{kg}^{1 / 3}$ on the specific direction. Regarding to the scaled impulse, the tendency is similar to that of peak overpressure, however, the range of angle that expanded is larger than that of peak overpressrure. Both isobaric lines are not symmetric on the line of $90^{\circ}$ and $270^{\circ}$. The blast wave is strong at former direction. The direction of ignition might affect the distribution.

In conclusion, the shape of the explosive and direction of ignition affected the blast pressure distribution. The blast pressure from $30^{\circ}$ to $80^{\circ}$ were high. These factsare important for safety consideration.

\section{References}

[1] R.A. Strehlow and W.E. Baker,The characterization and evaluation of accidental explosions,Prog. Energy Combut. Sci., 2 (1976) 27-60.

[2] H. Kleine, J. M. Dewey, K. Ohashi, T. Mizukaki and K. Takayama, Studies of the TNT equivalence of silver azide charges, Shock Waves, 13 (2003) 123-138. https://doi.org/10.1007/s00193-003-0204-3

[3] T. Homae, Y. Sugiyama, K. Wakabayashi, T. Matsumura, and Y. Nakayama, Blast wave mitigation from the straight tube by using water Part I -Small scale experiment-, Materials Sci. Forum, 910 (2018) 149-154. https://doi.org/10.4028/www.scientific.net/msf.910.149

[4] Y. Sugiyama, T. Homae, K. Wakabayashi, T. Matsumura, and Y. Nakayama, Numerical simulations of blast wave characteristics with a two-dimensional axisymmetric room model, Shock Waves,27 (2017) 615-622. https://doi.org/10.1007/s00193-016-0706-4

[5] T. Homae, K. Wakabayashi, T. Matsumura, and Y. Nakayama, Blast pressure distribution around a wall, Sci.Tech.Energetic Materials, 72 (2011) 155-160.

[6]Y. Sugiyama, T. Homae, K. Wakabayashi, T. Matsumura, and Y. Nakayama, Numerical simulations on the propagation of blast wave around a wall, Sci.Tech.Energetic Materials, 75 (2014) 162-168. 